\title{
Structure and function of mammalian cilia
}

\author{
Peter Satir $\cdot$ Søren T. Christensen
}

Accepted: 11 March 2008 / Published online: 26 March 2008

(C) Springer-Verlag 2008

\begin{abstract}
In the past half century, beginning with electron microscopic studies of $9+2$ motile and $9+0$ primary cilia, novel insights have been obtained regarding the structure and function of mammalian cilia. All cilia can now be viewed as sensory cellular antennae that coordinate a large number of cellular signaling pathways, sometimes coupling the signaling to ciliary motility or alternatively to cell division and differentiation. This view has had unanticipated consequences for our understanding of developmental processes and human disease.
\end{abstract}

Keywords Motile cilia $\cdot$ Primary cilia $\cdot$ Motility

Signaling $\cdot$ Sensory organelles $\cdot$ Ciliopathies

\section{Introduction}

Although in 1835, Purkinje and Valentin (1835) published the first treatise to include studies of mammalian cilia, the history of what may be considered modern research on mammalian cilia does not extend back more than a few years longer than the history of this journal. Motile mammalian cilia attracted the attention of microscopists before the advent of electron microscopy, because they moved and were obviously similar in this motion to protozoan cilia.

P. Satir

Department of Anatomy and Structural Biology,

Albert Einstein College of Medicine, Bronx, NY, USA

S. T. Christensen $(\square)$

Department of Biology,

Section of Cell and Developmental Biology,

University of Copenhagen, Copenhagen, Denmark

e-mail: stchristensen@bio.ku.dk
Nevertheless, several light microscope structures whose later fine structure appearance (and molecular biology) had little in common with cilia were nevertheless labeled cilia, including the stereocilia of the hair cells, which are in fact modified microvilli. In 1954, the electron microscope observations of Fawcett and Porter (1954) definitively characterized the $9+2$ pattern of the cytoskeleton, the axoneme, of motile mammalian and other cilia. They showed that this structure was enclosed by an extension of the cell membrane, the ciliary membrane. The axoneme of true cilia was always an extension of a basal body and related to the nine-fold construction of the centriole. By 1956, Porter (1957) and De Robertis (1956) realized that cilia were found as sensory structures in, for example mammalian photoreceptors, where the central pair of microtubules in the axoneme was absent, and the cilia were nonmotile, hence $9+0$. Finally, in the early 1960s, a number of electron microscopy studies showed that solitary $9+0$ cilia, now termed primary cilia, are present on many differentiated cells of tissues of the mammalian and vertebrate body, including kidney cells, fibroblasts, neurons and even Schwann cells (Barnes 1961; Sorokin 1962; Grillo and Palay 1963).

Although specific antibodies against detyrosinated and acetylated tubulin became available to detect primary cilia in immunofluorescence microscopy (Piperno et al. 1987; Cambray-Deakin and Burgoyne 1987), primary cilia were generally neglected as interesting cell organelles. The biggest problem was that primary cilia had no demonstrable function, even though considerable circumstantial evidence suggested that they were some form of chemical or mechanical sensor. On this basis, Poole et al. (1985) proposed that primary cilia of connective tissue cells functioned as a cellular cybernetic probe, or in current terminology, as a cellular global positioning system. Roth 
et al. (1988) then demonstrated that primary cilia on cultured kidney epithelial cells could be bent by flow, and Wheatley (1995) suggested that if primary cilia in renal epithelia did not function properly in sensing flow, there would be pathophysiological consequences. Nevertheless, many cell biologists dismissed the primary cilium as vestigial.

While early studies of mammalian cilia focused on showing that the mechanism and biochemistry of motility was virtually identical to that discovered in other cilia, more recent studies have examined the way that cilia are built. These studies have led to novel insights for both motile and nonmotile, including primary, cilia. All cilia can now be viewed as sensory cellular antennae that coordinate a plethora of cellular signaling pathways, and this view has had unanticipated consequences for our understanding of developmental processes and disease. A recent review of mammalian cilia structure and function covering many of the topics presented here, in further detail, is found in Satir and Christensen (2007).

\section{The mechanism of ciliary motility and primary ciliary dyskinesia}

The basic mechanism of ciliary motility is now reasonably well understood, although many details of how waveform is generated and propagated remain obscure. While much of the information about this mechanism was derived from protistan and invertebrate organisms (Satir 1985), the basic principles clearly apply to mammalian cilia of the respiratory and reproductive tract, and to brain ependymal cilia. An electron micrograph of cilia of the mouse oviduct is shown in Fig. 1.

Ciliary motility is caused by the relative sliding of the nine outer axonemal doublets, operating as two opposing sets, one (doublets 1-4) to produce the effective stroke (or principal bend in flagella), one (doublets 6-9) to produce the recovery stroke (or reverse bend), powered by ATP and a set of molecular motors, the axonemal dyneins. The axonemal dyneins are arranged in two sets of arms: the outer dynein arms (ODAs) consisting of two heavy-chain dynein isoforms in mammalian cilia, packaged with intermediate and light chains, with four identical ODAs, each aligned along 96-nm long doublet microtubule (Nicastro et al. 2006), and the more complex inner dynein arms (IDAs). The IDAs, more centrally located, consist of at least seven heterodimeric and monomeric heavy-chain dynein isoforms per $96 \mathrm{~nm}$. Generally, the ODAs and IDAs function together, but the ODAs principally regulate beat frequency, in part by cAMP-dependent phosphorylation of an ODA regulatory light chain, while the IDAs control beat form. Both

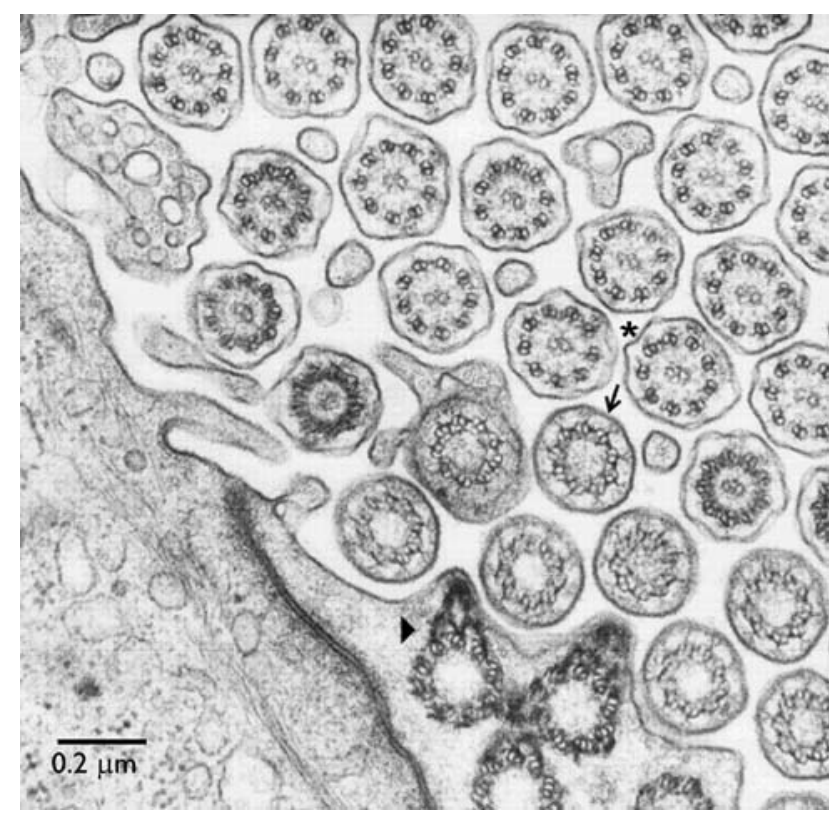

Fig. 1 Classic transmission electron micrograph of mouse oviduct cilia. Cross-sections show the $9+2$ axoneme of motile cilia (asterisk). The axoneme grows from a basal body, with a basal foot (arrowhead) pointing in the direction of the effective stroke. The transition zone between basal body and axoneme contains the ciliary necklace (arrow). (From Dirksen and Satir 1972, unpublished, with permission)

frequency and form change by changing the rate of sliding and the switching of activity between the doublet sets. Sliding is converted into propagated bending, most efficiently by control of IDA activity via phosphorylation and mechanical interaction involving the radial spoke, central pair complex.

In effect, the $9+2$ axoneme is a nanomachine composed of perhaps over 600 proteins in molecular complexes, many of which also function independently as nanomachines. The enzymes that control axonemal response, for example protein kinase A, are structurally part of the complexes. Strong support for this mechanism of ciliary motility comes from studies of paralyzed and other mutants of protistan cilia, where a structural defect and a corresponding genetic mutation can directly be correlated with a change in ciliary beat. Similarly, strong support for the applicability of the mechanism to human cilia comes from studies, mainly of ciliary structural defects and genetic mutations associated with impaired mucociliary function in the respiratory system, which produces sinusitis and bronchiectasis. The genetic diseases are now called primary ciliary dyskinesia (PCD). Clinical features of PCD, which include chronic rhinitis/ sinusitis, otitis media, male infertility and an increased incidence of hydrocephalus, point to physiological processes where ciliary motility is essential (Afzelius 2004). As might be anticipated, prominent causes of PCD are mutations that affect the assembly or function of the dynein 
arms-most commonly a mutation in DNAH5, a gene encoding a dynein heavy chain of the ODAs (Olbrich et al. 2002).

Any mutation affecting the ciliary beat mechanism will potentially produce PCD, depending on the severity of beat impairment. An instructive example is hydin, a component of the central pair projection complex that interacts with the radial spokes in the switching mechanism between doublet sets. Hydin-deficient flagella of Chlamydomonas become intermittently paralyzed, stopping for long times at the end of the effective or recovery strokes, where the direction of beat is reversed (Lechtreck and Witman 2007). Mice defective in Hydin develop hydrocephalus and die shortly after birth. Lechtreck et al. (2008) analyzed ciliary structure and motility in these mice. The central pair projection assigned to the hydin complex was missing. Mutant cilia were unable to bend properly and frequently stalled, implying that because central pair structure was defective, interactions between the central pair and the radial spokes that affected dynein arm switching were abnormal, and consequently ciliagenerated flow was impaired. Motility of tracheal cilia was also impaired. One would predict that human mutations in HYDIN are also likely to result in hydrocephalus or a form of PCD.

Many people diagnosed with PCD have situs inversus totalis that is reversal of left-right asymmetry of body organs such as the heart. This is now known to be produced, because fluid flow produced by motile cilia at the embryonic node is defective (Hirokawa et al. 2006). The usual flow pattern produced by the cilia is unidirectional right-to-left, and this activates a left-side-only gene cascade, probably by impinging on primary cilia at the left side of the node (McGrath et al. 2003). The nodal cilia are mainly missing the central pair of axonemal microtubules, hence, although motile, are often $9+0$, but with some unique ODA dynein isoforms (Supp et al. 1999). It is not known whether the response of the primary cilia at the node is to mechanical displacement (flow sensing) or if it involves morphogens produced in membrane parcels in the node (Tanaka et al. 2005). Morphogen-containing multimembrane vesicles are a feature of nodal response that could also apply respiratory and other ciliated epithelia, where flow occurs.

While mouse mutants or knockouts that are missing cilia develop situs inversus, hydin mutants do not show this phenotype, presumably because their motility is sufficient to generate appropriate nodal flow. In a similar way, by examining the PCD phenotype of mutations in various ciliary proteins, identified by proteomics, it should be possible to dissect details of the function of the protein in the mechanism of beat generation (Avidor-Reiss et al. 2004; Badano et al. 2006; Blacque et al. 2005).

\section{Principles of ciliogenesis}

A major breakthrough in the understanding of the importance of cilia in the body has come from the study of Chlamydomonas ciliogenesis. In that cell, the cilia are traditionally called 'flagella'. Ciliogenesis involves transport of materials into, along and out of the cilium, directly visualized as 'intraflagellar transport' or IFT (reviewed by Rosenbaum and Witman 2002). IFT requires molecular motors, several kinesins for anterograde transport and a specialized cytoplasmic dynein for retrograde transport. Coupling the transported cargo to the molecular motors that move along the outer doublets of the axoneme are two protein complexes (Cole 2003) comprising about 19 IFT proteins. Remarkably, this machinery is conserved almost universally wherever cilia are built, and orthologs of the motors and IFT proteins are found in sensory cilia and their derivatives, such as the mammalian photoreceptor (Baker et al. 2003) and in primary cilia, as well as in protistan motile cilia. However, motile cilia of mammalian tissues often assemble in mass (Gaillard et al. 1989; Dirksen 1991). Centriologenesis of hundreds of basal bodies occurs from a fibrogranular mass in the cytoplasm and cilia sprout from the cell surface. It is unclear whether classical IFT is the major mechanism of ciliogenesis in respiratory epithelium, or what modifications of the process occur.

Wheatley (1969), Fonte et al. (1971) and Archer and Wheatley (1971) studied ciliogenesis of primary cilia of established cell lines in vitro. Primary cilia grow when the cells become confluent and reach stationary phase. Cells resorb their cilia shortly before entering mitosis. Postmitotic cells reassemble primary cilia in $\mathrm{G} 1$ and maintain the cilium as the cells enter growth arrest $\left(\mathrm{G}_{0}\right)$ and undergo differentiation. In contrast to motile cilia, the primary cilium emanates uniquely from the distal end of the existing mother centriole of the centrosome, which migrates to the cell surface during growth arrest. Ciliogenesis by IFT is initiated while the centrosome is positioned at the Golgi apparatus near the nucleus, while extensive IFT and elongation of the cilium take place after docking of the nascent cilium at the cell surface, where microtubule pairs are quickly assembled to form the mature axoneme. Two recent and extensive reviews of the complex interactions between IFT particles, molecular motors, centrosomal proteins and other microtubule-associated proteins in ciliogenesis are presented in Pedersen et al. (2008) and Blacque et al. (2008).

\section{Ciliopathies}

The first human illness that could be linked to primary cilia in epithelial cells was polycystic kidney disease (PKD). One of the orthologs of IFT88 is the mammalian protein 
polaris mutated in a transgenic mouse $T g 737^{\circ r p k}$, which was a model for the study of PKD. Chlamydomonas IFT88 mutants are defective in ciliogenesis, and Pazour et al. (2000) demonstrated that, similarly, cilia of the mouse kidney were abnormally short or missing, which suggested that PKD might be a ciliary disease. This conclusion was strengthened by two succeeding discoveries. Mechanical deflection of kidney tubule epithelial cells induces an increase in intracellular calcium (Praetorius and Spring 2001), and the PKD proteins, polycystins 1 and 2 in $\mathrm{Ca}^{2+}$ signaling, localize to the ciliary membrane (Pazour et al. 2002; Yoder et al. 2002). Many other proteins whose functions are disrupted in cystic diseases, e.g. nephrocystins, have now been localized to the cilium or at the ciliary basal body, supporting the conclusion that the primary cilium in kidney tubules partly functions as a mechanosensor that, upon bending, activates a series of signaling pathways in the cilium to control development and homeostasis of the tissue (for reviews, see Yoder et al. 2007; Hildebrandt and Zhou 2007), as originally envisaged by Roth et al. (1988) and Wheatley (1995). Thus, even if primary cilia are present, unless these proteins are targeted appropriately to the cilia or the ciliary basal body, pathology develops.

It is now clear that the ciliary membrane is a privileged and specialized compartment for receptor signaling and that the polycystins are only one type of many membrane proteins that must be targeted to the primary cilium to function properly. Other carefully documented examples include the receptor tyrosine kinase PDGFR $\alpha$ (Schneider et al. 2005) and the patched receptors for hedgehog signaling (Rohatgi et al. 2007) that control cell growth and differentiation processes. Further, essential downstream components in PDGFR $\alpha$ and hedgehog signaling as well as signaling in neurotransmission, Wnt pathways, extracellular matrix interaction and osmolyte transportation uniquely localize to the cilium and/or to the ciliary centrosome (Gerdes et al. 2007; Corbit et al. 2008; for reviews, see Singla and Reiter 2006; Michaud and Yoder 2006; Christensen et al. 2007; Christensen and Ott 2007). In many of these signaling systems, the cellular response is initiated by receptor activation in the primary cilium. Deficiencies in placement of receptors and their immediate downstream signaling components in the cilium or at the ciliary base results in ciliopathy, including cystic kidney, pancreatic and liver diseases, retinitis pigmentosa, cancer, defective neurogenesis, BardetBiedl syndrome, polydactyly, anosmia and other developmental defects. In the $\mathrm{Tg} 737$ mouse, these defects lead to early perinatal death. As shown in Fig. 2, primary cilia with hedgehog receptor pathway signaling are found on human embryonic stem cells (Kiprilov et al. 2008), which suggests that ciliary signaling is involved in differentiation from the beginning of embryogenesis. In embryogenesis, signaling through the primary cilium is necessary for normal development, probably because such signaling regulates the balance between cell division, polarity, migration, differentiation and apoptosis for many tissues.

\section{Primary cilia in adult tissues}

Primary cilia persist in many differentiated cells, including kidney tubule epithelial cells, fibroblasts and neurons, after
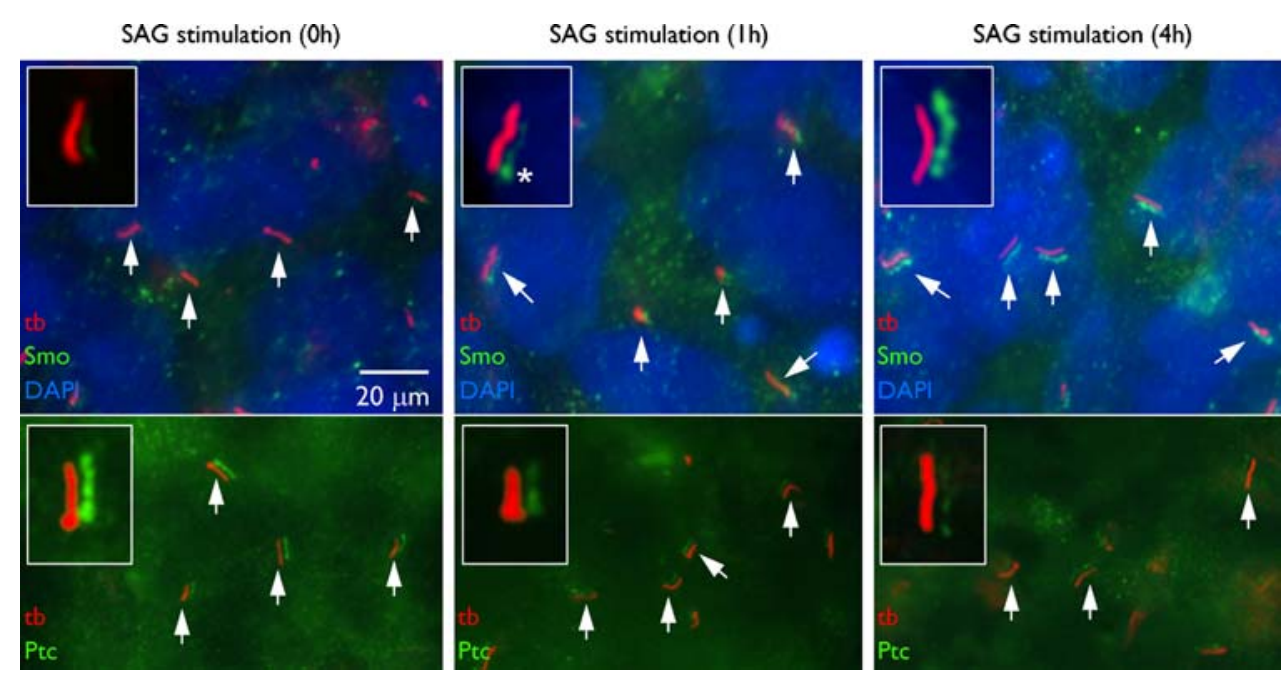

Fig. 2 Primary cilia of human embryonic stem cells. Immunofluoresence microscopy using acetylated $\alpha$ tubulin antibody (tb) reveals the presence of primary cilia (arrows) on human embryonic stem cells. In the absence of stimulation, the hedgehog receptor 'patched' (Ptc) colocalizes with the acetylated $\alpha$ tubulin all along the ciliary membrane. Red and green channels are displaced in the images to define colocal- ization more clearly. Nuclei are stained with DAPI (blue). Upon stimulation, as part of the signaling cascade, Ptc leaves the cilium and the smoothened receptor (Smo) enters to activate the hedgehog signaling cascade. Asterisk marks the ciliary base. (From Kiprilov et al. 2008, with permission, courtesy of The Journal of Cell Biology) 
organogenesis is complete and cell division rates fall. Presumably, as semipermanent structures, the cilia function as mechano- or chemosensors and as a cellular global positioning system to detect changes in the surrounding environment, to initiate cellular replacement after damage, for example. To test this hypothesis, techniques are being used to knock out primary cilia or ciliary proteins in specific tissues of adult organisms.

Davenport et al. (2007) have completed one of the first of these studies, using an inducible system in adult mice to disrupt IFT in several different ways, causing loss of primary cilia. Respiratory motile cilia are probably more stable and relatively unaffected. Surprisingly, when primary cilia are lost from all adult tissues, the devastating abnormalities and lethality seen after embryonic loss of cilia are not observed. PKD eventually develops a year after induction. The same delay has been reported by Piontek et al. (2007) after adult-specific knockout of polycystin-1. This delay correlates well with a greatly reduced rate of cell division and a different pattern of gene expression in the mature kidney and could explain the increase in human PKD with age. Such changes in cell proliferation rate may relegate the primary cilium of adult tissues to a less immediate role, involving long-term homeostasis of the tissue. Because signaling through primary cilia is coupled to cell cycle events, for example in PDGFR $\alpha \alpha$ signaling in fibroblasts, long-term disruption of ciliary signaling could be a factor in oncogenesis.

One adult tissue responds immediately to ciliary knockout however-that is nervous tissue, most specifically neurons in the hypothalamus. Knockout of all adult primary cilia in the mouse, or specifically only of primary cilia on POMC neurons, leads to hyperphagia-compulsive and excessive eating-leading to obesity. Obesity then causes numerous secondary defects resembling type II diabetes. These defects do not occur if the knockout mice are kept on a restricted diet. Eating behavior is regulated by the hormone leptin. These fndings suggest that the leptin receptor might be located in the membrane of the primary cilia of the POMC neurons.

The hypothalamic hormone somatostatin is a negative regulator of leptin. While leptin leads to reduced eating, somatostatin increases eating behavior. The somatostatin receptor sst 3 is localized to primary cilia in hypothalamic neurons (Stepanyan et al. 2007). The results of Davenport et al. (2007) suggest that, much like the patched and smoothened receptors work in hedgehog signaling, the leptin receptor and a somatostatin receptor could work in a Yin-Yang relationship within the POMC primary cilium (Satir 2007).

Whatever the precise cell biological explanation of the relationship between ciliary knockout and hyperphagia, the effect of knockout is behavioral. We might expect that mutations in other proteins of neuronal primary cilia could lead to other behavioral responses. There are reports that the rages that fueled the famous Hatfield-McCoy feud of Appalachia probably were driven by a family suffering from Von Hippel-Landau (VHL) syndrome. VHL protein controls ciliogenesis and is localized to cilia (Schermer et al. 2006). Perhaps a more ciliocentric view of neuronal activity affecting recurrent or addictive actions is warranted.

\section{Conclusions}

Beginning with important electron microscopic studies and culminating in immunolocalization combined with molecular genetic technology, much has been learned about motile, sensory and primary cilia in mammals. Defects in building the primary cilium or mutations in ciliary membrane or axonemal proteins lead to ciliopathies, important human diseases. The cilium has moved to a prominent place in studies of embryogenesis and tissue differentiation and maintenance. There are hints that the fundamental cell biology of cilia will also be important in oncogenesis, aging diseases and human behavioral disorders. The strides of the past half century in understanding this organelle have been impressive, and the promise of discovery in the next half century is compelling.

Acknowledgments We thank Raviraja Seetharam for helpful assistance. This work was supported in part by grants from the NIH to P.S. and from the Lundbeck Foundation (grant no. R9-A969) and The Danish Natural Science Research Council (grant no. 272-07-0530) to STC.

\section{References}

Afzelius BA (2004) Cilia-related diseases. J Pathol 204:470-477

Archer FL, Wheatley DN (1971) Cilia in cell-cultured fibroblasts. II. Incidence in mitotic and post-mitotic BHK 21-C13 fibroblasts. J Anat 109:277-292

Avidor-Reiss T, Maer AM, Koundakjian E, Polyanovsky A, Keil T, Subramaniam S, Zuker CS (2004) Decoding cilia function: defining specialized genes required for compartmentalized cilia biogenesis. Cell 117:527-539

Badano JL, Mitsuma N, Beales PL, Katsanis N (2006) The ciliopathies: an emerging class of human genetic disorders. Annu Rev Genomics Hum Genet 7:125-148

Baker SA, Freeman K, Luby-Phelps K, Pazour GJ, Besharse JC (2003) IFT20 links kinesin II with a mammalian intraflagellar transport complex that is conserved in motile flagella and sensory cilia. J Biol Chem 278:34211-34218

Barnes BG (1961) Ciliated secretory cells in the pars distalis of the mouse hypophysis. J Ultrastruct Res 5:453-467

Blacque OE, Cevik S, Kaplan OI (2008) Intraflagellar transport: from molecular characterisation to mechanism. Front Biosci 13:26332652

Blacque OE, Perens EA, Boroevich KA, Inglis PN, Li C, Warner A, Khattra J, Holt RA, Ou G, Mah AK, McKay SJ, Huang P, 
Swoboda P, Jones SJ, Marra MA, Baillie DL, Moerman DG, Shaham S, Leroux MR (2005) Functional genomics of the cilium, a sensory organelle. Curr Biol 15:935-941

Cambray-Deakin MA, Burgoyne RD (1987) Acetylated and detyrosinated alpha-tubulins are co-localized in stable microtubules in rat meningeal fibroblasts. Cell Motil Cytoskeleton 8:284-291

Christensen ST, Ott CM (2007) Cell signaling. A ciliary signaling switch. Science 317:330-331

Christensen ST, Pedersen LB, Schneider L, Satir P (2007) Sensory cilia and integration of signal transduction in human health and disease. Traffic 8:97-109

Cole DG (2003) The intraflagellar transport machinery of Chlamydomonas reinhardtii. Traffic 4:435-442

Corbit KC, Shyer AE, Dowdle WE, Gaulden J, Singla V, Reiter JF (2008) Kif3a constrains beta-catenin-dependent Wnt signalling through dual ciliary and non-ciliary mechanisms. Nat Cell Biol 10:70-76

Dirksen ER (1991) Centriole and basal body formation during ciliogenesis revisited. Biol Cell 72:31-38

Davenport JR, Watts AJ, Roper VC, Croyle MJ, van Groen T, Wyss JM, Nagy TR, Kesterson RA, Yoder BK (2007) Disruption of intraflagellar transport in adult mice leads to obesity and slow-onset cystic kidney disease. Curr Biol 17:1586-1594

De Robertis E (1956) Morphogenesis of the retinal rods; an electron microscope study. J Biophys Biochem Cytol 2(4, Suppl):209-218

Fawcett DW, Porter KR (1954) A study of the fine structure of ciliated epithelia. J Morphol 94:221-281

Fonte VG, Searls RL, Hilfer SR (1971) The relationship of cilia with cell division and differentiation. J Cell Biol 49:226-229

Gaillard DA, Lallement AV, Petit AF, Puchelle ES (1989) In vivo ciliogenesis in human fetal tracheal epithelium. Am J Anat 185:415428

Gerdes JM, Liu Y, Zaghloul NA, Leitch CC, Lawson SS, Kato M, Beachy PA, Beales PL, DeMartino GN, Fisher S, Badano JL, Katsanis N (2007) Disruption of the basal body compromises proteasomal function and perturbs intracellular Wnt response. Nat Genet 39:1350-1360

Grillo MA, Palay SL (1963) Ciliated Schwann cells in the autonomic nervous system of the adult rat. J Cell Biol 16:430-436

Hildebrandt F, Zhou W (2007) Nephronophthisis-associated ciliopathies. J Am Soc Nephrol 18:1855-1871

Hirokawa N, Tanaka Y, Okada Y, Takeda S (2006) Nodal flow and the generation of left-right asymmetry. Cell 125:33-45

Kiprilov EN, Awan A, Velho M, Clement CA, Byskov AG, Andersen CY, Satir P, Bouhassira EE, Christensen ST, Hirsch RE (2008) Human embryonic stem cells in culture possess primary cilia with hedgehog signaling machinery. J Cell Biol 180:897-904

Lechtreck KF, Delmotte P, Robinson ML, Sanderson MJ, Witman GB (2008) Mutations in hydin impair ciliary motility in mice. J Cell Biol 180:633-643

Lechtreck KF, Witman GB (2007) Chlamydomonas reinhardtii hydin is a central pair protein required for flagellar motility. J Cell Biol $176: 473-482$

McGrath J, Somlo S, Makova S, Tian X, Brueckner M (2003) Two populations of node monocilia initiate left-right asymmetry in the mouse. Cell 114:61-73

Michaud EJ, Yoder BK (2006) The primary cilium in cell signaling and cancer. Cancer Res 66:6463-6467

Nicastro D, Schwartz C, Pierson J, Gaudette R, Porter ME, McIntosh JR (2006) The molecular architecture of axonemes revealed by cryoelectron tomography. Science 313:944-948

Olbrich H, Häffner K, Kispert A, Völkel A, Volz A, Sasmaz G, Reinhardt R, Hennig S, Lehrach H, Konietzko N, Zariwala M, Noone PG, Knowles M, Mitchison HM, Meeks M, Chung EM, Hildebrandt F, Sudbrak R, Omran H (2002) Mutations in DNAH5 cause primary ciliary dyskinesia and randomization of left-right asymmetry. Nat Genet 30:143-144

Pazour GJ, Dickert BL, Vucica Y, Seeley ES, Rosenbaum JL, Witman GB, Cole DG (2000) Chlamydomonas IFT88 and its mouse homologue, polycystic kidney disease gene tg737, are required for assembly of cilia and flagella. J Cell Biol 151:709-718

Pazour GJ, San Agustin JT, Follit JA, Rosenbaum JL, Witman GB (2002) Polycystin-2 localizes to kidney cilia and the ciliary level is elevated in orpk mice with polycystic kidney disease. Curr Biol 12:R378-R380

Pedersen LB, Veland IR, Schrøder JM, Christensen ST (2008) Assembly of primary cilia. Dev Dyn (in press)

Piontek K, Menezes LF, Garcia-Gonzalez MA, Huso DL, Germino GG (2007) A critical developmental switch defines the kinetics of kidney cyst formation after loss of Pkd1. Nat Med 13:1490-1495

Piperno G, LeDizet M, Chang XJ (1987) Microtubules containing acetylated alpha-tubulin in mammalian cells in culture. J Cell Biol 104:289-302

Poole CA, Flint MH, Beaumont BW (1985) Analysis of the morphology and function of primary cilia in connective tissues: a cellular cybernetic probe? Cell Motil 5:175-193

Porter KR (1957) The submicroscopic morphology of protoplasm. Harvey Lect 51:175-228

Praetorius HA, Spring KR (2001) Bending the MDCK cell primary cilium increases intracellular calcium. J Membr Biol 184:71-79

Purkinje JE, Valentin G (1835) De phaenomeno generali et fundamentali motus vibratorii continui in membranis cum externis tum internis animalium plurimorum et superiorum et inferiorum ordinum obvii. In: Schulz A (ed) Commentatio Physiologica. Wratislaviae, p 96

Rohatgi R, Milenkovic L, Scott MP (2007) Patched1 regulates hedgehog signaling at the primary cilium. Science 317:372-376

Rosenbaum JL, Witman GB (2002) Intraflagellar transport. Nat Rev Mol Cell Biol 3:813-825

Roth KE, ReiderCL, Bowser SS (1988) Flexible substratum for viewing cells from the side: some in vivo properties of primary $(9+0)$ cilia in cultured kidney epithelia. J Cell Sci 89:457-466

Satir P (1985) Switching mechanisms in the control of ciliary motility. In: Satir P (ed) Modern cell biology. Alan R. Liss, Inc., New York, pp 1-46

Satir P (2007) Cilia biology: stop overeating now! Curr Biol 17:R963R965

Satir P, Christensen ST (2007) Overview of structure and function of mammalian cilia. Annu Rev Physiol 69:377-400

Schermer B, Ghenoiu C, Bartram M, Müller RU, Kotsis F, Höhne M, Kühn W, Rapka M, Nitschke R, Zentgraf H, Fliegauf M, Omran H, Walz G, Benzing T (2006) The von Hippel-Lindau tumor suppressor protein controls ciliogenesis by orienting microtubule growth. J Cell Biol 175:547-554

Schneider L, Clement CA, Teilmann SC, Pazour GJ, Hoffmann EK, Satir P, Christensen ST (2005) PDGFR $\alpha \alpha$ signaling is regulated through the primary cilium in fibroblasts. Curr Biol 15:18611866

Singla V, Reiter JF (2006) The primary cilium as the cell's antenna: signaling at a sensory organelle. Science 313:629-633

Sorokin S (1962) Centrioles and the formation of rudimentary cilia by fibroblasts and smooth muscle cells. J Cell Biol 15:363-377

Supp DM, Brueckner M, Kuehn MR, Witte DP, Lowe LA, McGrath J, Corrales J, Potter SS (1999) Targeted deletion of the ATP binding domain of left-right dynein confirms its role in specifying development of left-right asymmetries. Development 126:5495-5504

Stepanyan Z, Kocharyan A, Behrens M, Koebnick C, Pyrski M, Meyerhof W (2007) Somatostatin, a negative-regulator of central leptin action in the rat hypothalamus. J Neurochem 100:468-478 
Tanaka Y, Okada Y, Hirokawa N (2005) FGF-induced vesicular release of Sonic hedgehog and retinoic acid in leftward nodal flow is critical for left-right determination. Nature 435:172-177

Wheatley DN (1969) Cilia in cell-cultured fibroblasts: I. On their occurrence and relative frequencies in primary cultures and established cell lines. J Anat 105:351-362

Wheatley DN (1995) Primary cilia in normal and pathological tissues. Pathobiology 63:222-238
Yoder BK (2007) Role of primary cilia in the pathogenesis of polycystic kidney disease. J Am Soc Nephrol 18:1381-1388

Yoder BK, Hou X, Guay-Woodford LM (2002) The polycystic kidney disease proteins, polycystin-1, polycystin-2, polaris, and cystin, are co-localized in renal cilia. J Am Soc Nephrol 13:2508-2516 